\title{
Continuous Decline in Myocardial Infarction and Heart Failure Hospitalizations During the First 12 Months of the COVID-19 Pandemic in Israel
}

\author{
Gil Lavie ${ }^{1,4, \dagger},{ }^{*}$, Yael Wolff Sagy ${ }^{1}+$, Moshe Hoshen ${ }^{1}$, Walid Saliba ${ }^{2,4}$, Moshe Y. Flugelman ${ }^{3,4}$
}

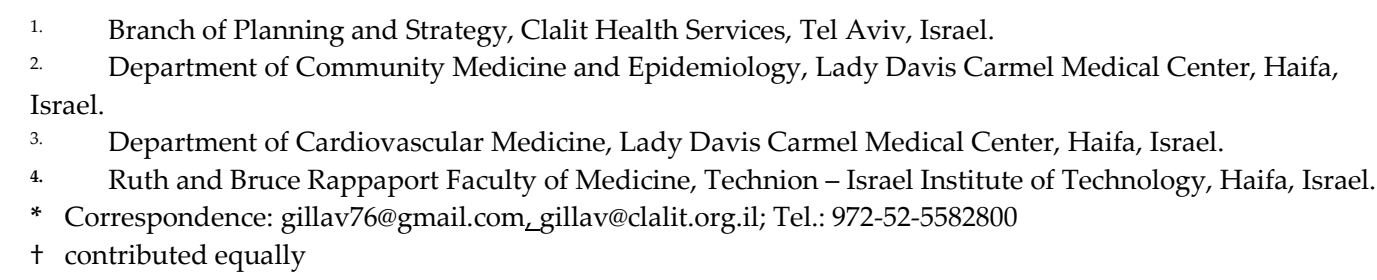

\begin{abstract}
Background. A decline in cardiovascular hospitalizations was observed during the initial phases of the COVID-19 pandemic. We examine the continued effect of the pandemic on cardiovascular hospitalizations and the associated mortality rates during the first year of the pandemic in Israel. Methods. We conduct a retrospective cohort study using the data of Clalit Health Services, the largest healthcare organization in Israel. We divide the Corona year into six periods (three lockdowns and three post-lockdowns) and compare the incidence rates of cardiovascular hospitalizations and their 30-day mortality during each period to the previous three years. Results. The number of non-STEMI hospitalizations during the first year of the pandemic was $13.7 \%$ lower than the average of the previous three years (95\% CI 11\%-17\%); STEMI hospitalizations were $15.7 \%$ lower (95\% CI 13\%-19\%); and CHF hospitalizations were $23.9 \%$ lower (95\%, CI 21\%-27\%). No significant differences in 30-day mortality rates were observed for AMI patients during most of the periods, whereas the annual 30-day all-cause mortality rate of CHF was $23 \%$ higher. Conclusions. Hospitalizations for AMI and CHF were significantly lower during the first year of the pandemic relative to 2017-9. Mortality rates were higher in the case of CHF patients but not in the case of AMI patients, possibly due a change in the clinical acuity of patients arriving at the hospitals. We conclude that targeted public health messaging should be implemented, together with proactive monitoring in order to identify residual disability in patients that may have received non-optimal treatment during the pandemic.
\end{abstract}

Keywords: COVID-19 pandemic; Acute myocardial infarction; Congestive heart failure; Cardiovascular hospitalizations

\section{Introduction}

A significant decline in hospitalizations for acute myocardial infarction (AMI) and congestive heart failure ( $\mathrm{CHF}$ ), which can be attributed to social containment mandates, was reported worldwide during the initial phases of the COVID-19 pandemic. ${ }^{1-4}$ This reduction in cardiovascular-related hospitalization may have had significant effects on public health, with respect to both out-of-hospital mortality and long-term complications of myocardial infarction, such as fatal arrhythmia and disabling heart failure ${ }^{5}$. The evidence from various other studies for the continuing effect of the pandemic on hospitalization trends is mixed ${ }^{6-8}$. In this study, we sought to examine the impact of the COVID-19 pandemic during its first 12 months in Israel on AMI and CHF hospitalization rates and on 30-day all-cause mortality rates, relative to the previous three years. 


\section{Methods}

\subsection{Study design and population}

A retrospective cohort study was carried out which included all AMI and CHF hospitalizations among Clalit Health Services (CHS) members aged 18 and older for the period March 14th 2017-March 13 th 2021. CHS is the largest HMO in Israel (where health insurance is universal and mandatory), with a membership of $52 \%$ of the population (4.8 million individuals). $\mathrm{CHS}$ has a central computerized database in which all members have a nearly complete digital record. All hospitals in Israel are required to report patient diagnoses to the payer HMO. Thus, the dataset is virtually complete for the CHS share of the market, which can be considered representative of the total population.

\subsection{Study variables}

AMI cases were divided into STEMI (ICD 9 codes: 4100x, 4101x, 4102x, 4103x, 4104x, 4105x, 4106x, 4108x) and non-STEMI (ICD 9 codes: 4107x, 4109x) while CHF cases were identified as ICD-9 code 428x. In addition, the dataset provided the demographic characteristics of all CHS members aged 18 years and older, including sex, date of birth (in order to calculate age, which was subsequently categorized into $60+$ and under 60 ), ethnicity (general, ultra-Orthodox Jewish or Arab, according to the ethnic classification of the member's primary clinic), and background diagnoses, which were used to calculate the Charlson comorbidity index (CCI; categorized into up to 4 or 5 and above 4 or 5), a measure of comorbidity load.

\subsection{Comparison Periods}

Years were defined as beginning on March $14^{\text {th }}$ and ending on March $13^{\text {th }}$, such that the sample period was 2017-18 to 2020-2021, where 2020-21 is the "Corona year". Incidence rates were calculated at the weekly level. The comparison periods were defined according to the three lockdowns as follows (according to calendar weeks): first lockdown (week 10-15); post-first lockdown (week 16-36); second lockdown (week 37-41); post-second lockdown (week 42-51); third lockdown (week 52-6); and post-third lockdown (week 7-10). (Note that week 52 and week 1 of the following year represent incomplete weeks.)

\subsection{Data Analysis}

Mean weekly incidence rates of hospitalization for STEMI, non-STEMI and CHF were calculated per 1,000 person-years. These rates were then compared between the Corona year and the average of the three baseline years for each period. Case fatality rates (CFR), defined as the proportion of all-cause death within 30 days of hospital admission (whether in- or out-of-hospital), were calculated for each period, and the OR for 30-day mortality in the Corona year relative to previous years was estimated using generalized estimating equations (GEE) in a logistic regression, adjusting for age, sex and CCI. All calculations were performed using R software version 4.1.1, together with two basic packages: "RODBC" for data extraction from the data warehouse and "gee". Significance was set at $p<0.05$. In view of the descriptive nature of the analysis, no adjustment was made for multiple comparisons. The CHS Institutional Ethics Board approved the study with a waiver of informed consent.

\section{Results}

The breakdown of total cases between the three baseline years and the Corona year is as follows: STEMI - 9,562 patients (yearly average of 3,187) vs. 2,758 patients; nonSTEMI - 22,060 patients (yearly average of 7,353) vs. 6,509 patients; and CHF - 108,856 patients (yearly average of 36,285 ) vs. 28,352 patients. No differences in incidence were detected according to age, sex, ethnicity or CCI (Table 1). Therefore, only overall changes in incidence are reported. We present rates of STEMI, non-STEMI and CHF 
hospitalization per 1,000 person-years for each period during the Corona year vs the three baseline years.

Table 1. Population and patient characteristics, Corona year and average of three baseline years.

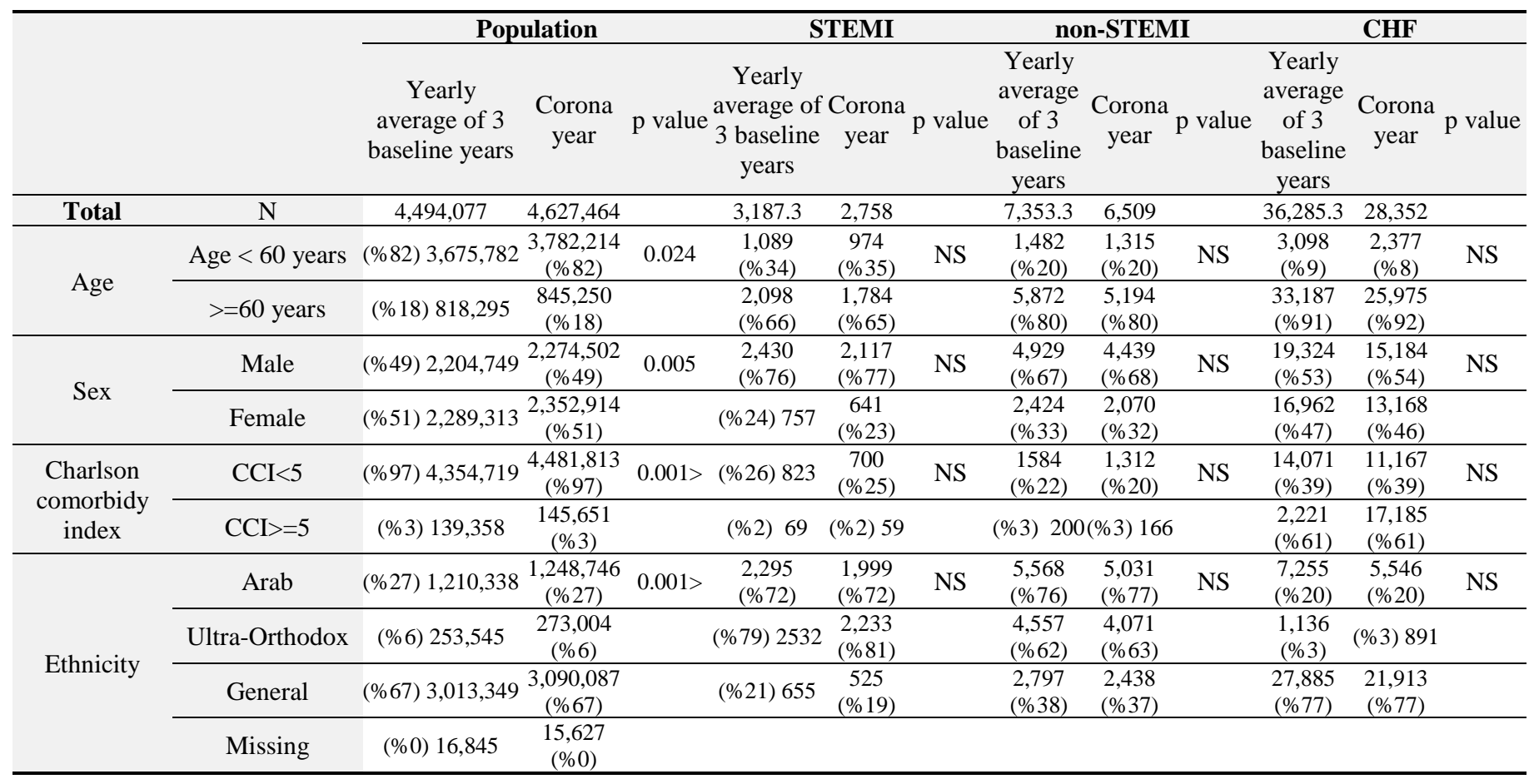

The overall STEMI incidence rate during the Corona year was 0.89 cases per 1,000 person-years, representing a decline of $15.7 \%$ (95\% CI: 13\%, 19\%) relative to the baseline years. STEMI incidence rates were lower throughout the year and in particular during the third lockdown (24\% decline relative to the baseline years; 95\% CI: $-21,-27 \%$ ) and during the post-third lockdown period ( $22 \%$ decline relative to the baseline years; $95 \% \mathrm{CI}:-19$, 25\%) (see Table 2a and Figure 1a). Non-STEMI incidence during the Corona year was 2.11 cases per 1,000 person-years, representing a decline of $13.7 \%$ (95\% CI: $11 \%, 17 \%)$ relative to the baseline years. When examining each period separately, and in contrast to STEMI incidence, the decline in non-STEMI incidence was not significant in any of the periods: following a significant reduction in incidence of $19 \%$ relative to baseline during the first lockdown (95\% CI: $-16 \%,-22 \%)$, non-STEMI incidence rates rebounded to the vicinity of the baseline rates during the post-first lockdown period and the second lockdown period. Thereafter, incidence rates dropped by $25 \%$ in the post-second lockdown period (95\% CI: $-22 \%,-28 \%)$; by $34 \%$ in the third lockdown period (95\% CI: $-31 \%,-37 \%)$, and by $19 \%$ in the post-third lockdown period (95\% CI: $-16 \%,-22 \%$ ) (see Table $2 \mathrm{~b}$ and Figure $1 \mathrm{~b}$ ).

Table 2. a: STEMI incidence by period for the Corona year and the baseline years.

\begin{tabular}{|c|c|c|c|c|c|c|c|c|}
\hline STEMI & & $\begin{array}{c}\begin{array}{c}\text { First } \\
\text { lockdown }\end{array} \\
\end{array}$ & $\begin{array}{l}\text { Post-first } \\
\text { lockdown }\end{array}$ & $\begin{array}{c}\text { Second } \\
\text { lockdown }\end{array}$ & $\begin{array}{l}\text { Post-second - } \\
\text { lockdown }\end{array}$ & $\begin{array}{c}\text { Third } \\
\text { lockdown }\end{array}$ & $\begin{array}{l}\text { Post-third } \\
\text { lockdown }\end{array}$ & Total \\
\hline & Calendar weeks & 10 to 15 & 16 to 36 & 37 to 41 & 42 to 51 & 52 to 6 & 7 to 10 & \\
\hline \multirow{3}{*}{ Baseline years } & $\mathrm{N}$ of cases & 1,068 & 3,905 & 845 & 1,556 & 1,239 & 949 & 9,562 \\
\hline & total person-years & $1,022,379$ & $3,578,329$ & 851,983 & $1,703,966$ & $1,192,777$ & 851,983 & $9,031,021$ \\
\hline & Incidence per 1,000 PY & 1.04 & 1.09 & 0.99 & 0.91 & 1.04 & 1.11 & 1.06 \\
\hline \multirow{3}{*}{ Corona year } & $\mathrm{N}$ of cases & 314 & 1153 & 266 & 449 & 323 & 253 & 2,758 \\
\hline & total person-years & 349688 & 1223909 & 291407 & 582814 & 407970 & 291407 & 3088913 \\
\hline & Incidence per 1,000 PY & 0.90 & 0.94 & 0.91 & 0.77 & 0.79 & 0.87 & 0.89 \\
\hline & o reduction & \%14- & \%13.7- & \%8- & \%15.6- & \%23.8- & $\% 22-$ & \%15.7- \\
\hline \multicolumn{2}{|c|}{ 95\% CI (upper, lower) } & $-11 \%,-17 \%$ & $-11 \%,-17 \%$ & $-5 \%,-11 \%$ & $-13 \%,-19 \%$ & $-21 \%,-27 \%$ & $-19 \%,-25 \%$ & $\begin{array}{l}-13 \% \\
-19 \% \\
\end{array}$ \\
\hline
\end{tabular}


Table 2. b: Non-STEMI incidence by period during the Corona year and baseline years.

\begin{tabular}{|c|c|c|c|c|c|c|c|c|}
\hline Non-STEMI & & First lockdown & $\begin{array}{l}\text { Post-first } \\
\text { lockdown }\end{array}$ & $\begin{array}{c}\text { Second } \\
\text { lockdown }\end{array}$ & $\begin{array}{l}\text { Post-second - } \\
\text { lockdown }\end{array}$ & $\begin{array}{c}\text { Third } \\
\text { lockdown }\end{array}$ & $\begin{array}{l}\text { Post-third } \\
\text { lockdown }\end{array}$ & Total \\
\hline & Calendar weeks & 10 to 15 & 16 to 36 & 37 to 41 & 42 to 51 & 52 to 6 & 7 to 10 & \\
\hline \multirow{3}{*}{ Baseline years } & $\mathrm{N}$ of cases & 2,640 & 8,201 & 1,989 & 4,027 & 2,964 & 2,239 & 22,060 \\
\hline & total person-years & $1,022,379$ & $3,578,329$ & 851,983 & $1,703,966$ & $1,192,777$ & 851,983 & $9,031,021$ \\
\hline & Incidence per $1,000 \mathrm{PY}$ & 2.58 & 2.29 & 2.33 & 2.36 & 2.48 & 2.63 & 2.44 \\
\hline \multirow{5}{*}{ Corona year } & $\mathrm{N}$ of cases & 735 & 2778 & 670 & 1036 & 667 & 623 & 6,509 \\
\hline & total person-years & 349,688 & $1,223,909$ & 291,407 & 582,814 & 407,970 & 291,407 & $3,088,913$ \\
\hline & Incidence per $1,000 \mathrm{PY}$ & 2.10 & 2.27 & 2.30 & 1.78 & 1.63 & 2.14 & 2.11 \\
\hline & $\%$ reduction & \%18.6- & $\% 1-$ & $\% 1.5-$ & $\% 24.8-$ & \%34.2- & \%18.6- & $\% 13.7-$ \\
\hline & 95\% CI (upper, lower) & $-16 \%,-22 \%$ & $2 \%,-4 \%$ & $1 \%,-5 \%$ & $-22 \%,-28 \%$ & $-31 \%,-37 \%$ & $-16 \%,-22 \%$ & $-11 \%,-17 \%$ \\
\hline
\end{tabular}

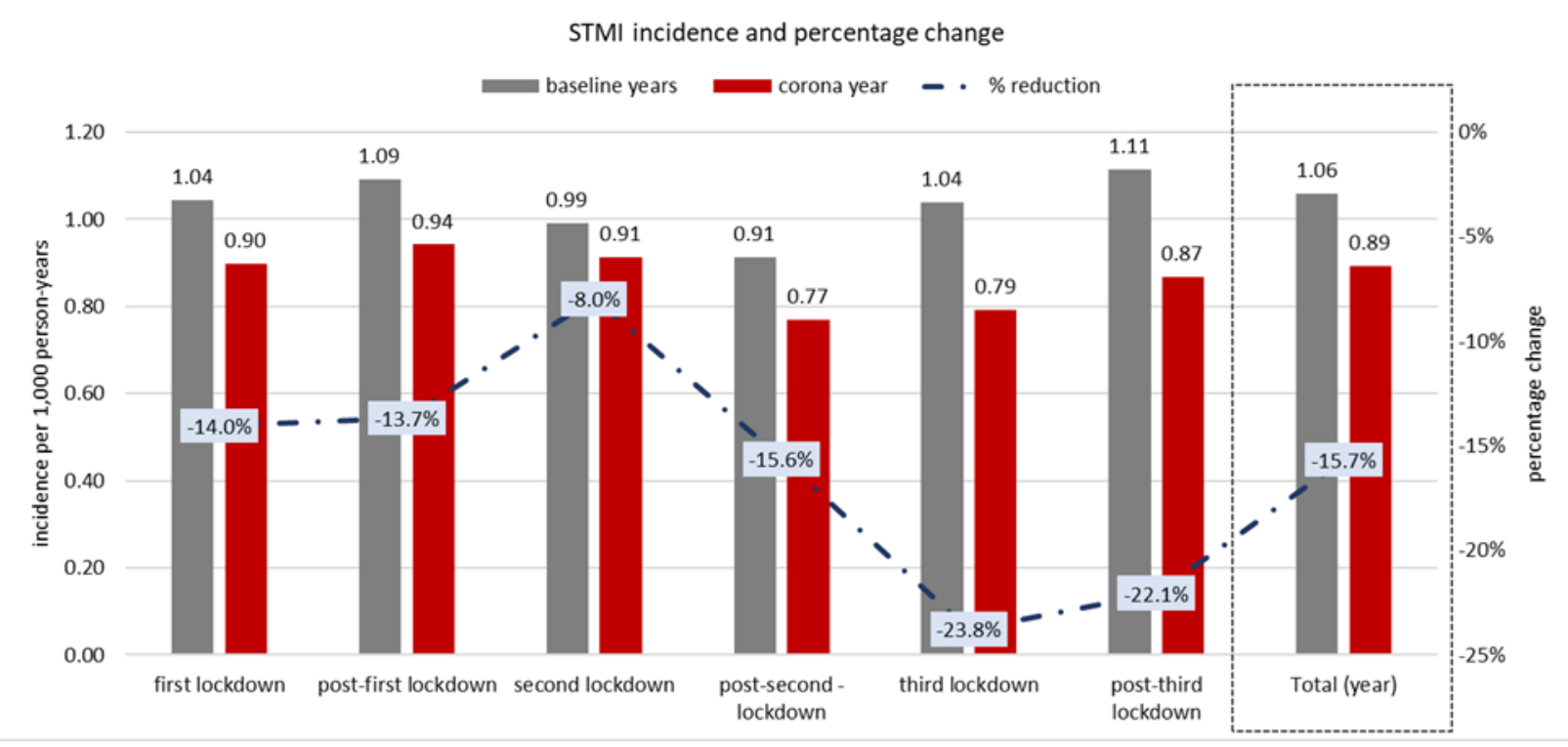

Figure 1. a: STEMI incidence by period during the Corona year and the average of preceding three years, and percentage change (hospitalization cases per 1,000 person-years).

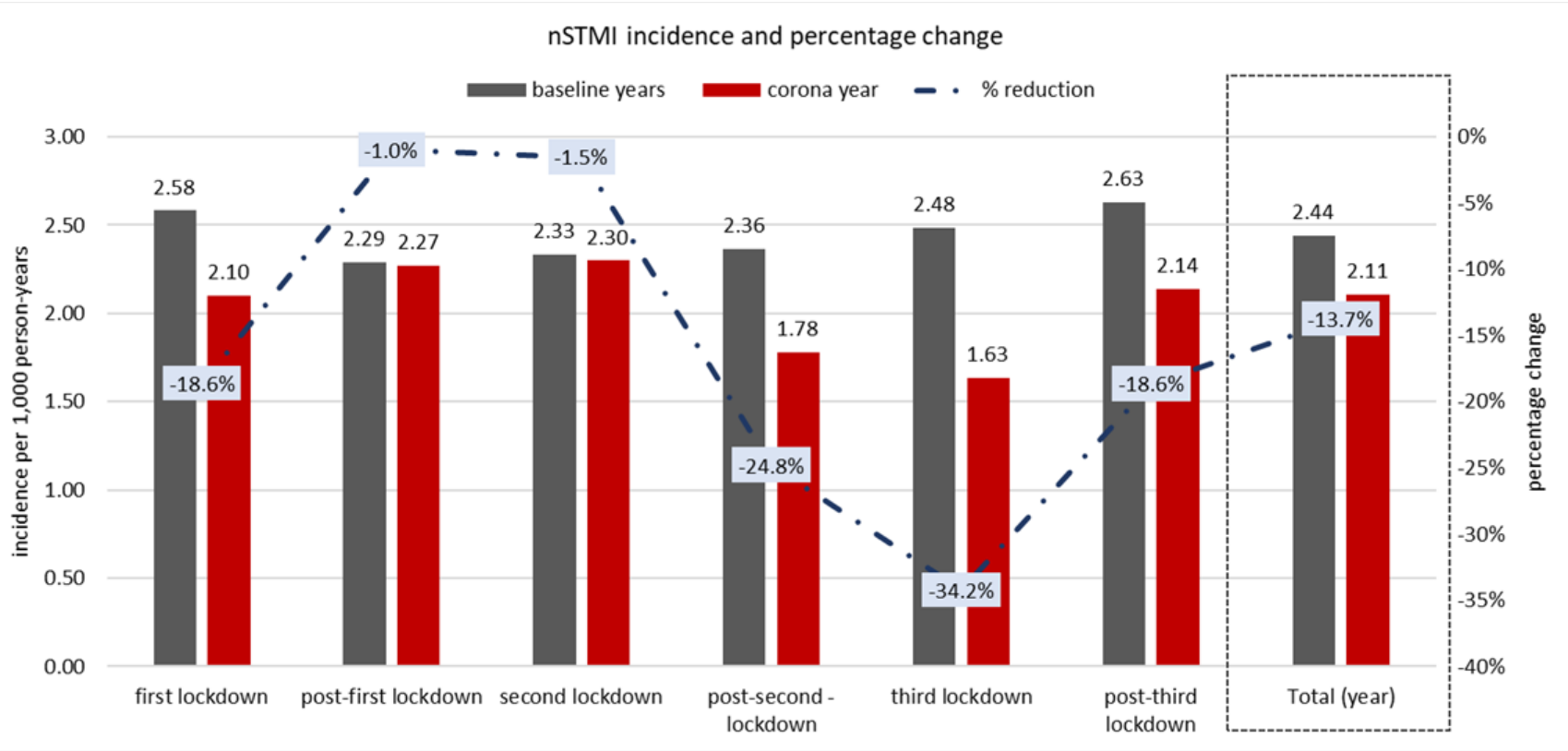

Figure 1. b: non-STEMI incidence by period during the Corona year and the average of preceding three years, and percentage change (hospitalization cases per 1,000 person-years). 
The overall CHF incidence during the Corona year was 9.18 cases per 1,000 personyears, representing a decline of $23.9 \%$ (95\% CI: $-21 \%$, $-27 \%)$ relative to the baseline years. When examining each period separately, the reduction in $\mathrm{CHF}$ incidence remained similar throughout the year, ranging from $17 \%$ in the post-first lockdown period and second lockdown period (95\% CI: $-14 \%,-20 \%)$ up to a peak of 33\% (95\% CI: $-30 \%,-36 \%)$ in the third lockdown period (see Table 2c and Figure 1c).

Table 2. c: CHF incidence by period during the Corona year and baseline years.

\begin{tabular}{|c|c|c|c|c|c|c|c|c|}
\hline CHF & & first lockdown & $\begin{array}{l}\text { post-first } \\
\text { lockdown }\end{array}$ & $\begin{array}{c}\text { second } \\
\text { lockdown }\end{array}$ & $\begin{array}{l}\text { post-second - } \\
\text { lockdown }\end{array}$ & $\begin{array}{c}\text { third } \\
\text { lockdown }\end{array}$ & $\begin{array}{l}\text { post-third } \\
\text { lockdown }\end{array}$ & Total (year) \\
\hline & calendar weeks & 10 to 15 & 16 to 36 & 37 to 41 & 42 to 51 & 52 to 6 & 7 to 10 & \\
\hline \multirow{3}{*}{$\begin{array}{l}\text { Baseline } \\
\text { years }\end{array}$} & $\mathrm{N}$ of cases & 14167 & 42191 & 9058 & 17771 & 14295 & 11374 & 108856 \\
\hline & total person-years & $\begin{array}{c}102237 \\
9\end{array}$ & 3578329 & 851983 & 1703966 & 1192777 & 851983 & 9031021 \\
\hline & Incidence per $1,000 \mathrm{PY}$ & 13.86 & 11.79 & 10.63 & 10.43 & 11.98 & 13.35 & 12.05 \\
\hline \multirow{3}{*}{$\begin{array}{c}\text { Corona } \\
\text { year }\end{array}$} & $\mathrm{N}$ of cases & 3359 & 12018 & 2568 & 4387 & 3281 & 2739 & 28352 \\
\hline & total person-years & 349688 & 1223909 & 291407 & 582814 & 407970 & 291407 & 3088913 \\
\hline & Incidence per $1,000 \mathrm{PY}$ & 9.61 & 9.82 & 8.81 & 7.53 & 8.04 & 9.40 & 9.18 \\
\hline & $\%$ reduction & \%30.7- & \%16.7- & \%17.1- & \%27.8- & \%32.9- & \%29.6- & $\% 23.9-$ \\
\hline \multicolumn{2}{|c|}{ 95\% CI (upper, lower) } & $-28 \%,-34 \%$ & $-14 \%,-20 \%$ & $-14 \%,-20 \%$ & $-25 \%,-31 \%$ & $-30 \%,-36 \%$ & $-27 \%,-33 \%$ & $-21 \%,-27 \%$ \\
\hline
\end{tabular}

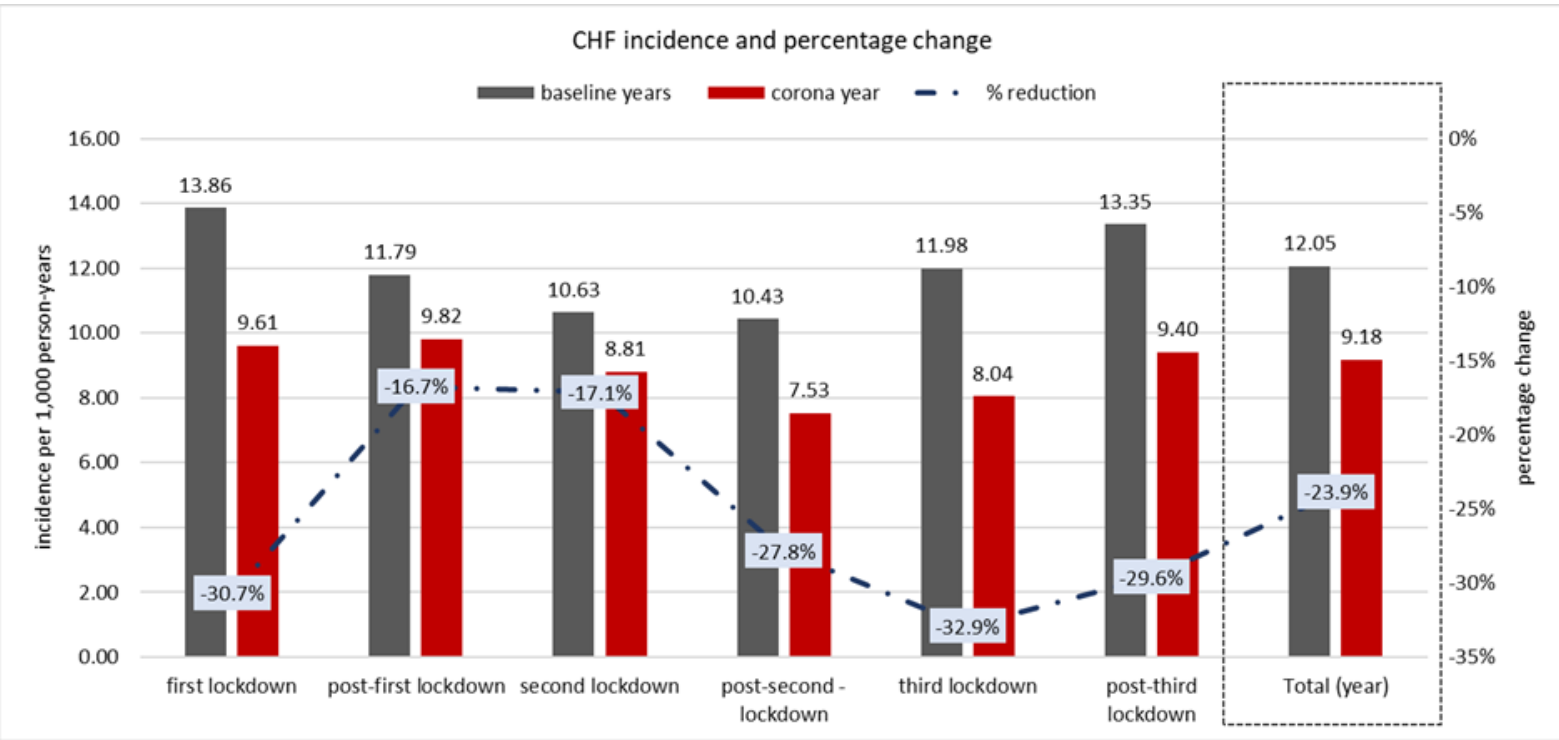

Figure 1. c: CHF incidence by period during the Corona year and the average of preceding three years, and percentage change (hospitalization cases per 1,000 person-years.)

The increase in the CFR for the entire year was significant in the case of CHF (adjusted OR-adjOR 1.226 95\% CI: 1.172-1.282) and borderline significant in the case of nonSTEMI (adjOR 1.104 95\% CI: 1.001-1.217), but was not significant for STEMI (adjOR 1.091 95\%CI: 0.934-1.275), though there was inter-period variability.

When examining each period separately, a significant increase in the CFR among STEMI patients was observed only in the post-second lockdown period (adjOR 1.702 95\% CI: 1.155-2.508) (Figure 3a). In the case of non-STEMI patients, an increase in the CFR was observed only during the second lockdown period relative to the baseline years (adjOR 1.451 95\%CI: 1.064-1.978) (Figure 3b). In contrast to the AMI patients, patients admitted with $\mathrm{CHF}$ during the Corona year had higher case fatalities relative to the baseline years in all periods, except for the post-second lockdown period, with a peak during the second lockdown period (adjOR 1.713 95\% CI: 1.486-1.974) (Figure 3c). 
STEMI: adjusted* OR for case-fatality

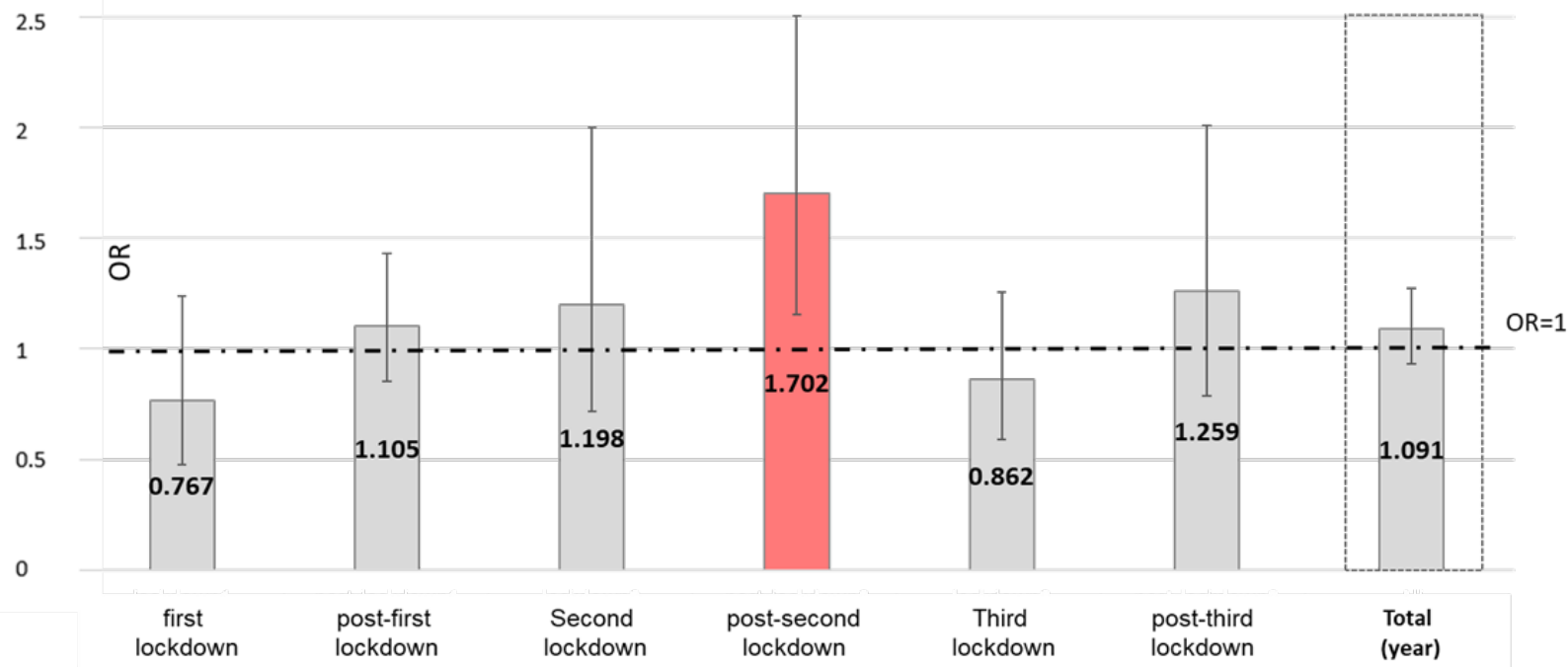

Figure 2. a: STEMI adjusted case fatality rate during the Corona year vs the corresponding baseline periods in the preceding three years (OR from GEE logistic models, adjusted for age, sex, and CCI).

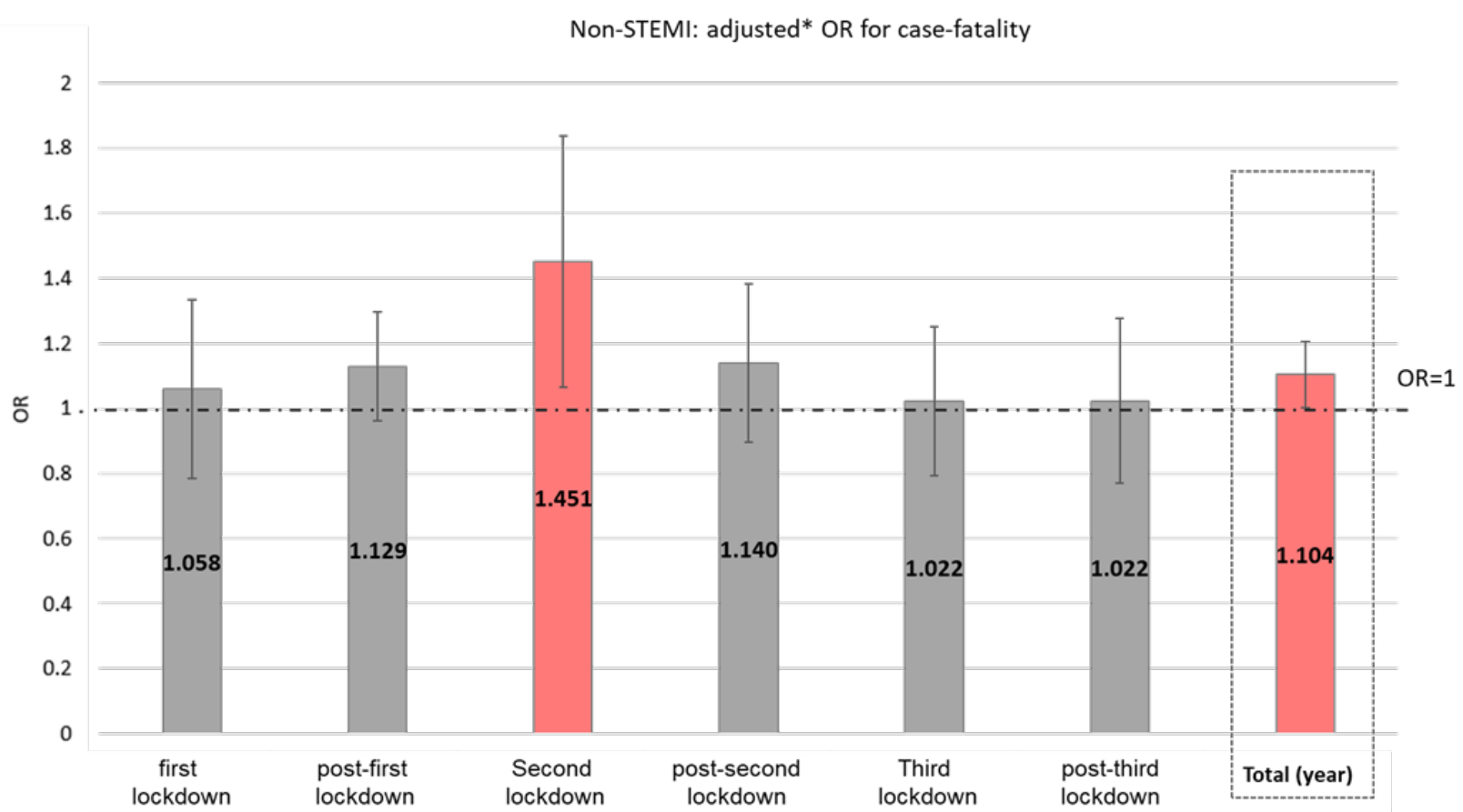

Figure 2. b: Non-STEMI adjusted case fatality rate during the Corona year vs the corresponding baseline periods in the preceding three years (OR from GEE logistic models, adjusted for age, sex, and CCI). 


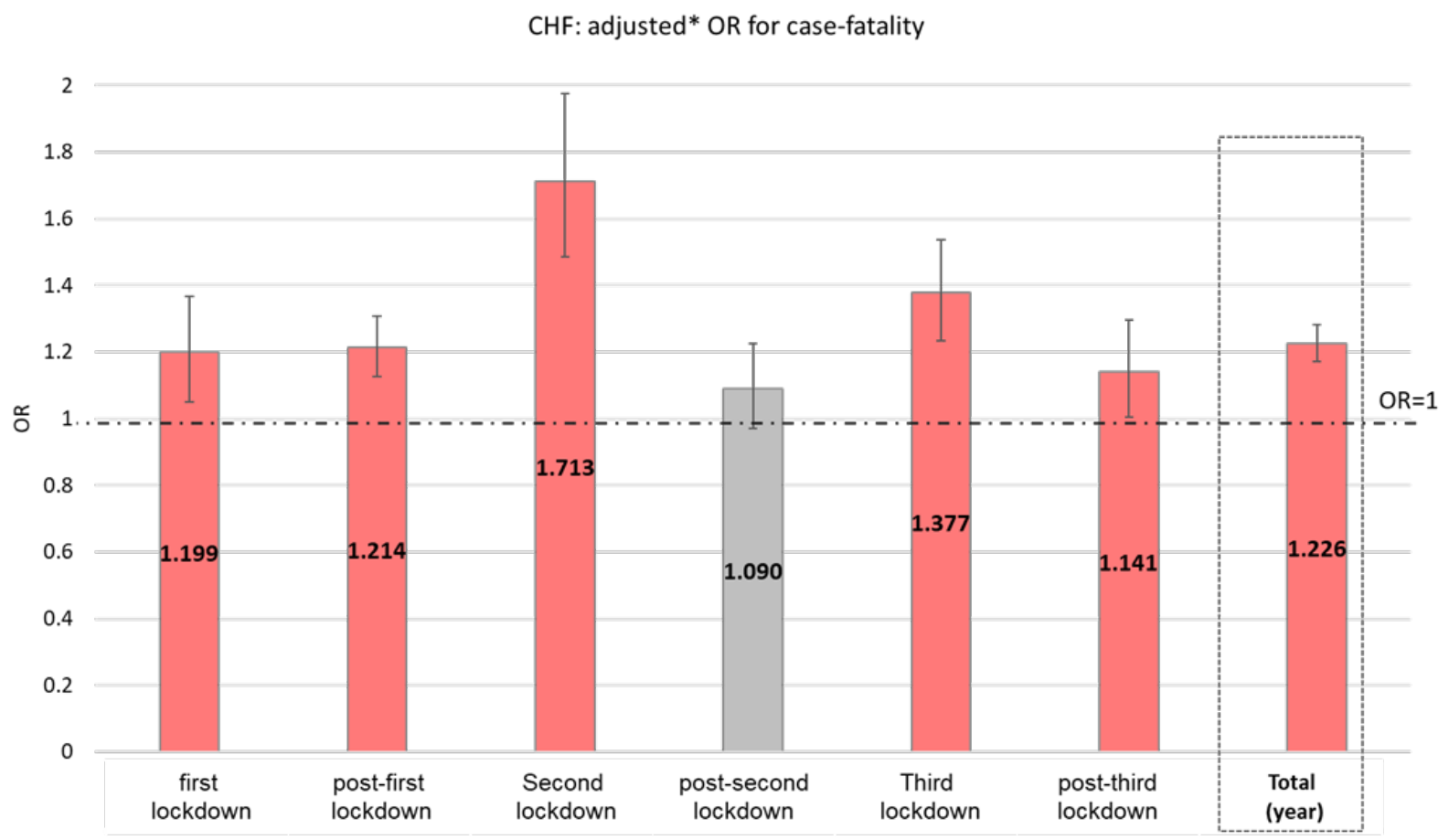

Figure 2. c: CHF adjusted case fatality rate during the Corona year vs the corresponding baseline periods in the preceding three years (OR from GEE logistic models, adjusted for age, sex, and CCI).

\section{Discussion}

The current study demonstrates a significant and continuous decline in AMI and CHF hospitalization rates during the first year of the COVID-19 pandemic in Israel relative to the previous three years. We have demonstrated an annual decline of $15.7 \%$ in STEMI hospitalization (CI 95\%: 13\%, 19\%), a 13.7\% decline in non-STEMI hospitalization (CI 95\%: 11\%, 17\%) and a 23.9\% decline in CHF hospitalization (CI 95\%: 21\%, 27\%). While 30-day all-cause mortality rates among both STEMI and non-STEMI patients during the Corona year were quite similar to those among AMI patients in the previous years, mortality rates among CHF patients were 22.6\% higher (adjOR 1.226 95\%CI: 1.172-1.282), and the differences were statistically significant for most of the study periods.

There are researchers who claim that an actual decrease in the incidence of cardiovascular events during the pandemic cannot be ruled out since staying at home may have reduced $\mathrm{AMI}^{9,10}$ by limiting the exposure to external triggers of acute coronary events (such as pollution and workplace stress). However, it is widely believed that the indirect health effects of the pandemic reduced the consumption of cardiovascular hospitalization services ${ }^{11}$. Though the reasons for the reduction are not fully understood, they are mostly attributed to the imposition of social isolation, "shelter-in-place orders", and similar regulations during the surges in COVID-19 cases. Their goal was to encourage the public not to come to medical centers and to avoid unnecessary healthcare use in order to reduce transmission of the virus and ensure that hospital capacity could accommodate surges in COVID-19 cases $^{13,14}$. Certain patient populations also reported forgoing medical care, mainly owing to fear of SARS-CoV2 infection ${ }^{15}$, or in an attempt to mitigate the burden on the healthcare system ${ }^{16}$, in line with public health messages communicated by governments.

Various studies worldwide showed a different pattern of cardiovascular hospitalization rates later in the pandemic. In the $\mathrm{UK}^{6}$, a study showed similar declines in AMI and $\mathrm{CHF}$ hospitalizations during the first and second lockdown periods. The researchers suggest that the public were fearful of visiting hospitals and concluded that clear public 
messaging is necessary to prevent further unintended consequences of social distancing. Another study in the UK examined CHF hospitalization rates during the three COVID surges and lockdowns and found that despite the public health messages and healthcare reconfiguration, the admission rates remained significantly reduced throughout the entire COVID pandemic, with no differences between the three lockdown periods examined ${ }^{17}$. In contrast to these studies and in line with our findings, a study in Denmark ${ }^{8}$ demonstrated a further reduction in cardiovascular hospitalization rates during the second COVID surge, though it was smaller in magnitude than during the first lockdown. The researchers concluded that declines in cardiovascular admission rates may be preventable during COVID-19 case surges. A study carried out in the US7 using data of Kaiser Permanente Northern California showed no significant decline in AMI hospitalization during the second lockdown, as compared to the decline observed in the first lockdown. According to the researchers, this may reflect changing patient attitudes during the COVID-19 pandemic or the success of health system and public health campaigns to reassure patients about the safety of seeking emergency care when necessary?

The variability in the rates of decline in cardiovascular hospitalization rates across countries during the pandemic is evidence of the multiplicity of underlying factors. This variability can be attributed to the non-uniform intensity of the pandemic across countries and variation in the stringency of lockdown measures. It may also be due to public-healthrelated factors, such as differences in the structure and infrastructure of the local health care system; its ability to provide a preventative community response and hospitalization substitute; and variation in both the messages conveyed to the public regarding the importance of coming to the ER during a medical emergency and in the public's level of trust. The variation in the rate of decline in cardiovascular hospitalization rates across the various surges shown in the Danish and American studies supports the existence of a reversible component that can be minimized, despite the surges in COVID-19 cases. Therefore, the decline in seeking medical attention may not be driven directly by lockdowns per se, but rather by other factors such as fear of the virus or lack of trust in authorities $^{8}$. According to our findings, the decline in the rate of cardiovascular hospitalizations weakened during the second surge (with non-STEMI hospitalizations returning to their normal level), but reached its peak during the third surge. Therefore, and as demonstrated in other studies, the level of COVID morbidity alone, which rose in Israel from the first surge to the second and from the second to the third, is not a sufficient explanation for this phenomenon. These findings highlight the importance of intensifying public health messaging and reinforcing the public's confidence in the safety of coming to the hospital for emergency treatment in future COVID surges, in order to mitigate the decline in cardiovascular hospitalization and the under-treatment of cardiovascular conditions.

As in other studies ${ }^{17-19}$, we did not identify a significant rise in the 30-day mortality rates during most of the periods among AMI patients relative to previous years; however, we did find higher rates among CHF patients during almost all of the periods examined ${ }^{20}$. The shift of system resources in favor of treating COVID-19 patients at the expense of providing optimal care to other patients is unlikely to explain our findings, in view of the fact that the burden of COVID-19 morbidity and the shift in system resources increased over the course of the year, while AMI and $\mathrm{CHF}$ mortality rates did not demonstrate a constant upward trend. Therefore, a possible explanation for our findings and for the difference in mortality rates between AMI patients and CHF patients may be the difference in the characteristics of patients arriving at the ER and in the severity of their condition. While AMI is an acute and urgent diagnosis, which usually has a clear presentation and results in a rapid evacuation to the ER and hospitalization, CHF often has a more moderate and gradual presentation and may be dealt with in the community without an ER admission. Furthermore, as a result of these differences some of the individuals suffering from CHF exacerbation may have delayed going to hospital and eventually arrived in a more severe condition due to their reluctance to be in a hospital environment ${ }^{21}$. Therefore, 
it is possible that while the severity of AMI among patients arriving at the hospital did not change significantly during the pandemic, the CHF patients (whose number declined more than that of AMI patients) included a higher proportion of more critical patients who were characterized by a higher mortality rate. As found in other studies ${ }^{20,22,23}$, we believe that cardiovascular patients who did arrive at the ER during the Corona year received care at a similar level to that in the preceding non-pandemic years and that the quality of care was not compromised during the pandemic. The increased rates of mortality among CHF patients may reflect a change in mix of acuity among CHF patients arriving at the ER ${ }^{17,24}$. Similar findings of reduced CHF hospitalizations accompanied by an increase in 30-day mortality were reported by Shoaib et al. ${ }^{20}$.

In conclusion, our findings show a continuous decline in cardiovascular hospitalizations during the Corona year and a possible change in the mix of CHF patients. This reinforces the need for public health officials to encourage patients not to delay necessary medical care during future COVID surges and to initiate proactive long-term follow-up in order to identify the health consequences of the possible sub-optimal treatment of acute cardiovascular conditions during the Corona year.

This study has several strengths. First, we were able to examine weekly AMI and $\mathrm{CHF}$ hospitalization rates in a large integrative healthcare organization during the first year of the pandemic in Israel. Second, the length of the sample period, the extent of population coverage and the availability of computerized up-to-date data gathered in both inpatient and community settings provided a unique dataset of AMI and CHF hospitalizations. Finally, the accessibility and completeness of the data made it possible to examine 30-day all-cause mortality.

Limitations: The main limitation of the study is the lack of data on out-of-hospital cardiovascular mortality among individuals who refrained from going to the hospital. This limited our ability to fully analyze the pandemic's potential collateral harm in discouraging individuals from seeking necessary treatment, even in emergencies. Furthermore, lack of data on concomitant COVID-19 infection in AMI and CHF patients may potentially affect the rates of cardiovascular hospitalization, in view of the possible increase in cardiac enzymes during a COVID-19 infection. The study is also limited to hospitals in Israel, and the results may not be generalizable to other countries.

\section{Conclusions}

We carried out a large retrospective cohort study which demonstrated a significant reduction in AMI and CHF hospitalizations during the first year of the pandemic in Israel, relative to the preceding three years. Although 30-day all-cause mortality did not change significantly for AMI patients, it increased among CHF patients hospitalized during the first year of the pandemic, possibly due to a worsening in the clinical acuity of patients arriving at the hospitals, rather than lower standards of care. The study's findings have a number of implications for future surges of COVID-19 morbidity: practitioners and public health officials should encourage patients not to delay essential medical care that cannot be managed in other settings ${ }^{14}$. The public should understand the importance of seeking guidance and emergency care for acute cardiovascular conditions, and possible barriers to necessary medical care should be addressed with targeted public health messaging in order to prevent collateral cardiovascular damage ${ }^{12}$. Further research is needed in order to proactively monitor the potential consequences over time of forgoing or deferring care of acute cardiovascular conditions during the Corona year.

\section{Supplementary Materials:}

Author Contributions: Gil Lavie - Conceptualization, Supervision, Writing - original draft, Writing - review \& editing, Yael Wolff Sagy - Data curation, Methodology, Formal analysis, Writing - review \& editing, Moshe Hoshen - Data curation, Formal analysis, Walid Saliba - Formal analysis, Methodology, Writing - review \& editing, Moshe Y. Flugelman - Conceptualization, Writing - original draft, Writing - review \& editing. 
Funding: No funding was provided.

Institutional Review Board Statement: Not applicable.

Informed Consent Statement:

Data Availability Statement: Data are available upon request.

Acknowledgments:

Conflicts of Interest: The authors report no relationships that could be construed as a conflict of interest

\section{References}

1. Matthew D Solomon, Edward J McNulty, Jamal S Rana et al. The Covid-19 Pandemic and the Incidence of Acute Myocardial infarction. N Engl J Med 2020 Aug 13;383(7):691-693

2. Infarctionhttps://www.who.int/news/item/01-06-2020-covid-19-significantly-impacts-health-services-for-noncommunicablediseases

3. Pessoa-Amorim, Christian F Camm, Parag Gajendragadkar et al. Admission of patients with STEMI since the outbreak of the COVID-19 pandemic: a survey by the European Society of Cardiology. Guilherme Eur Heart J Qual Care Clin Outcomes 2020 Jul 1;6(3):210-216

4. Ovidio De Filippo, Fabrizio D'Ascenzo, Filippo Angelini et al. Reduced Rate of Hospital Admissions for ACS during Covid-19 Outbreak in Northern Italy. N Engl J Med 2020 Jul 2;383(1):88-89.

5. Marion M Mafham, Enti Spata, Raphael Goldacre. COVID-19 pandemic and admission rates for and management of acute coronary syndromes in England. Lancet 2020 Aug 8;396(10248):381-389

6. Jianhua Wu, Mamas A Mamas, Mark A de Belder et al. Second Decline in Admissions With Heart Failure and Myocardial Infarction During the COVID-19 Pandemic. J Am Coll Cardiol 2021 Mar 2;77(8):1141-1143

7. Matthew D Solomon, Mai Nguyen-Huynh, Thomas K Leong et al. Changes in Patterns of Hospital Visits for Acute Myocardial Infarction or Ischemic Stroke During COVID-19 Surges. JAMA 2021 Jul 6;326(1):82-84.

8. Daniel Mølager Christensen, Jawad Haider Butt, Emil Fosbøl et al. Nationwide cardiovascular disease admission rates during a second COVID-19 lockdown. Am Heart J. 2021 Nov; 241: 35-37.

9. M. A. Mohammad,S. Koul,C. P. Gale,J. Alfredsson et al. The association of mode of location activity and mobility with acute coronary syndrome: a nationwide ecological study. J Intern Med 2021 Feb;289(2):247-254.

10. Francesco Sofi, Monica Dinu, GianPaolo Reboldi et al. Worldwide differences of hospitalization for ST-segment elevation myocardial infarction during COVID-19: A systematic review and meta-analysis. Int J Cardiol 2022 Jan 15;347:89-96.

11. Eugenia Wong, Wayne Rosamond, Mehul D Patel et al. Statewide declines in myocardial infarction and stroke emergency department visits during COVID-19 restrictions in North Carolina. Am J Emerg Med 2021 Jul 22;S0735-6757(21)00590-8.

12. Charlotte M Roy, E Brennan Bollman, Laura M Carson et al. Assessing the indirect effects of COVID-19 on healthcare delivery, utilization and health outcomes: a scoping review. Eur J Public Health 2021 Jul 13;31(3):634-640.

13. Ankeet S Bhatt, Alea Moscone, Erin E McElrath et al. Fewer Hospitalizations for Acute Cardiovascular Conditions During the COVID-19 Pandemic. J Am Coll Cardiol 2020 Jul 21;76(3):280-288.

14. Molly M Jeffery, Gail D'Onofrio, Hyung Paek et al. Trends in Emergency Department Visits and Hospital Admissions in Health Care Systems in 5 States in the First Months of the COVID-19 Pandemic in the US. JAMA Intern Med 2020 Oct 1;180(10):13281333.

15. Jonathan L Ciofani, Daniel Han, Usaid K Allahwala et al. Internet search volume for chest pain during the COVID-19 pandemic. Am Heart J. 2021 Jan; 231: 157-159.

16. Mark É Czeisler, Kristy Marynak, Kristie E N Clarke et al. Delay or Avoidance of Medical Care Because of COVID-19-Related Concerns - United States, June 2020. MMWR Morb Mortal Wkly Rep 2020 Sep 11;69(36):1250-1257.

17. Irfan A Rind, Antonio Cannata, Benedict McDonaugh et al. Patients hospitalised with heart failure across different waves of the COVID-19 pandemic show consistent clinical characteristics and outcomes. Int J Cardiol 2022 Mar 1;350:125-129.

18. Fabrizio D'Ascenzo, Ovidio De Filippo, Andrea Borin et al. Impact of COVID-19 pandemic and infection on in hospital survival for patients presenting with acute coronary syndromes: A multicenter registry. Int J Cardiol 2021 Jun 1;332:227-234.

19. Jawad H Butt, Lauge Østergaard, Thomas A Gerds et al. The association between cardiovascular disease admission rates and the coronavirus disease 2019 lockdown and reopening of a nation: a Danish nationwide cohort study. Eur Heart J Qual Care Clin Outcomes 2022 Jan 5;8(1):14-22.

20. Ahmad Shoaib, Harriette G C Van Spall, Jianhua Wu et al. Substantial decline in hospital admissions for heart failure accompanied by increased community mortality during COVID-19 pandemic. Eur Heart J Qual Care Clin Outcomes 2021 Jul 21;7(4):378-387.

21. Vishal N Rao, Michelle D Kelsey, Anita M Kelsey et al. Acute cardiovascular hospitalizations and illness severity before and during the COVID-19 pandemic. Clin Cardiol 2021 May;44(5):656-664.

22. Antonio Cannatà, Daniel I Bromage, Irfan A Rind et al. Temporal trends in decompensated heart failure and outcomes during COVID-19: a multisite report from heart failure referral centres in London. Eur J Heart Fail 2020 Dec;22(12):2219-2224 
23. Karl Heinrich Scholz, Björn Lengenfelder, Christian Thilo et al. Impact of COVID-19 outbreak on regional STEMI care in Germany. Clin Res Cardiol 2020 Dec;109(12):1511-1521.

24. Antonio Cannata, Samuel A Watson, Allen Daniel et al. Impact of the COVID-19 pandemic on in-hospital mortality in cardiovascular disease: a meta-analysis. Eur J Prev Cardiol 2021 Jul 23;zwab119. 